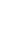

\title{
Modular-Based Psychotherapy (MoBa) versus Cognitive Behavioural Therapy (CBT) for patients with comorbid depression and a history of childhood maltreatment: Study protocol for a
} randomised controlled feasibility trial

Moritz Elsaesser ${ }^{1}$, Sabine Herpertz ${ }^{2}$, Hannah Piosczyk ${ }^{1}$, Carolin Jenkner ${ }^{3}$, Martin Hautzinger $^{4}$ \& Elisabeth Schramm ${ }^{1^{*}}$

1 Department of Psychiatry and Psychotherapy, University Medical Center University of Freiburg, Faculty of Medicine, University of Freiburg, Freiburg, Germany

2 Department of General Psychiatry, Center for Psychosocial Medicine, Medical Faculty, Heidelberg University, Heidelberg, Germany

${ }^{3}$ Clinical Trials Unit, Faculty of Medicine, University Medical Center, University of Freiburg, Freiburg, Germany

4 Department of Psychology, Clinical Psychology, and Psychotherapy, Eberhard Karls University of Tübingen, Tübingen, Germany

Word count: 3998

*Correspondence:

\section{Prof. Dr. Elisabeth Schramm}

Department of Psychiatry and Psychotherapy

University Medical Center - University of Freiburg, Hauptstraße 5

DE-79104 Freiburg (Germany)

$\square$ elisabeth.schramm@uniklinik-freiburg.de 


\section{Abstract}

Introduction: In depression treatment, most patients do not reach response or remission with current psychotherapeutic approaches. Major reasons for individual non-response are interindividual heterogeneity of etiological mechanisms and pathological forms, and a high rate of comorbid disorders. Personalised treatments targeting comorbidities as well as underlying transdiagnostic mechanisms and factors like early childhood maltreatment may lead to better outcomes. A Modular-Based Psychotherapy (MoBa) approach provides a treatment model of independent and flexible therapy elements within a systematic treatment algorithm to combine and integrate existing evidence-based approaches. By optimally tailoring module selection and application to the specific needs of each patient, MoBa has great potential to improve the currently unsatisfying results of psychotherapy as a bridge between disorder-specific and personalised approaches.

Methods and analysis: In a randomized controlled feasibility trial (RCT), N=70 outpatients with episodic or persistent major depression, comorbidity and childhood maltreatment are treated in 20 individual sessions with MoBa or standard Cognitive Behavioural Therapy (CBT) for depression. The three modules of MoBa focus on deficits associated with early childhood maltreatment: the systems of negative valence, social processes, and arousal. According to a specific questionnaire-based treatment algorithm, elements from Cognitive Behavioural Analysis System of Psychotherapy (CBASP), Mentalization-Based Psychotherapy (MBT) and/or Mindfulness (MBCT) are integrated for a personalised modular procedure.

As a proof of concept, this trial will provide evidence for the feasibility and efficacy (posttreatment and six month follow-up) of a modular add-on approach for patients with depression, comorbidities and a history of childhood maltreatment. Crucial feasibility aspects include targeted psychopathological mechanisms, selection (treatment algorithm), sequence and application of modules, as well as training and supervision of the study therapists.

Ethics and dissemination: This study obtained approval from independent Ethics Committees. All findings will be disseminated broadly via peer-reviewed articles in scientific journals and contributions to national and international conferences. 
64

Trial registration: German Clinical Trials Register (www.drks.de): DRKS00022093.

Keywords: Modular-Based Psychotherapy | Cognitive Behavioural Therapy | Depression | Childhood Maltreatment | Treatment Algorithm | Personalised | Randomized Controlled Trial | Cognitive Behavioural Analysis System of Psychotherapy | Mentalization-Based Psychotherapy | Mindfulness

\section{Abstract Summary}

\section{Strengths and limitations of this study}

- This is the first study to investigate the feasibility of a Modular-Based Psychotherapy (MoBa) approach for patients with comorbid depression and a history of childhood maltreatment.

- Besides feasibility, this RCT will prove initial evidence for the efficacy of MoBa and generate pilot data for a subsequent multicentre confirmatory trial.

- If successful, clinicians will be provided with an evidence-based treatment algorithm to combine and integrate available treatment modules systematically instead of ad libitum eclecticism.

- Using Cognitive Behavioural Therapy (CBT) as control condition represents a strong comparator for a rigorous evaluation with a high generalizability to the clinical reality.

- Since no a priori values are established, the algorithm cut-offs used here are based on general population means of self-rated questionnaires. 


\section{Introduction}

Until recently, depressive disorders have been predominantly conceptualized and researched with a focus on the primary diagnosis. This has led to the development of several disorderspecific approaches such as the Cognitive Behavioural Therapy (CBT) [1] and the Interpersonal Psychotherapy (IPT) [2]. While these approaches (among others) have proven efficacy in unipolar major depression, there is a large proportion of patients who do not respond (more than 50\%) or do not reach full remission (about two thirds) with first line treatment [3], even when the procedure is in accordance with treatment guidelines [4,5]. Major reasons for individual non-response and non-remission include interindividual heterogeneity of etiological mechanisms of depression and high rates of comorbid disorders of up to $80 \%$ in clinical and epidemiological studies [6-8]. Particularly anxiety disorders and Cluster C personality disorders are highly prevalent in Major depressive disorder (MDD) [9]. These comorbid disorders typically predict poorer treatment outcomes for MDD [10-13] or longer time to remission [14].

\section{Childhood maltreatment}

One major transdiagnostic factor associated with cognitive, emotional, behavioural and interpersonal dysfunctions common to a wide range of disorders is childhood maltreatment (CM). CM has most frequently been operationalized based on the Childhood Trauma Questionnaire (CTQ) [15], defined as onset reported before the age of 18 and meeting the criterion of at least "moderate to severe" on one of the five trauma subtypes (emotional abuse, emotional neglect, physical abuse, physical neglect, sexual abuse). In depressive disorders, CM is highly prevalent ( 46\%) [16], especially in early-onset and persistent depression with up to $80 \%[17,18]$. An emerging body of evidence suggests a significant relationship between emotional maltreatment (abuse and/or neglect) in particular and depression [19-22]. Maltreated individuals are 2.7 to 3.7 times more likely to develop depression in adulthood, have an earlier depression onset and are twice as likely to develop a chronic or treatment-resistant course [16]. CM was also associated with an elevated risk for comorbid disorders $[23,18]$. Treated with psychotherapy and pharmacotherapy, the probability of non-response is 1.9 times 
110 higher in depressed patients with early trauma compared to those without [16]. Taken together,

111 study results indicate that interpersonal trauma exposure complicates the treatment of 112 depression and reduces the impact of traditional cognitive therapy or treatments such as 113 psychoeducation, TAU, or pharmacotherapy [24]. However, some approaches like 114 Mindfulness Based Cognitive Therapy (MBCT) [25] or the Cognitive Behavioural Analysis 115 System of Psychotherapy (CBASP) [26-28] show promising results in the subgroup of 116 depressed patients with $\mathrm{CM}$.

\section{Impact of childhood maltreatment on social and emotional functioning}

118 A growing body of evidence links interpersonal trauma in both youth and adults to difficulties 119 in social and emotional functioning [24]. Among other sequelae, CM usually results in marked avoidance behaviour [9] with negative social consequences and in concomitant retardation of emotional maturational growth [28,29]. These deficits are also expressed in terms of social threat hyperresponsivity (i.e. being highly sensitive to social rejection and anxiously expecting, readily perceiving, and overreacting to it) [30-33], social stress and avoidance behaviour $[34,35]$, lack of empathy and theory-of-mind [36-38] and emotional dysregulation $[39,40]$. These emotional and social dysfunctions are mediated in common brain circuits for emotion and salience regulation, fear, and mentalising, suggesting that abnormalities in these functional pathways may be induced by CM $[41,42]$. Despite these severe consequences of $\mathrm{CM}$ and their important implications for treatment, disorder-specific approaches for depression such as CBT or IPT do not specifically address the role of CM and the affected dimensions of functioning.

\section{Personalised Treatments}

This calls for personalised treatments that target both comorbidities as well as underlying mechanisms and factors, which are central to the development and maintenance of psychological disorders. One of the challenges in the development of personalised approaches is to select treatment modules for targeted dysfunctions and to determine whether and in which sequence to combine them with standard treatment. In daily practice, it is left to the clinical 
137 judgement and expertise of the therapist to address the patient's individual needs and

138 comorbidities by adding various therapeutic strategies to the disorder-specific interventions.

139 However, this choice of add-on strategies is not backed up by empirical evidence and thus 140 hardly conveyable to usual clinical practice in a systematic way [43]. Driven by these concerns, 141 there has been growing consensus that a novel approach is needed in the way we classify,

142 formulate, treat, and prevent depression and other mental disorders [44,45]. Insel and Cuthbert

143 [46] postulated the concept of Research Domain Criteria (RDoC) to move "toward a new 144 classification system" of studying and validating transdiagnostic, dimensional constructs since 145 psychiatric diagnosis seem to be no longer optimal as long as they remain restricted to 146 symptoms and signs. The transdiagnostic procedure focuses on identifying the common and 147 core maladaptive temperamental, cognitive, emotional, interpersonal and behavioural 148 characteristics that underpin a broad array of diagnostic presentations [47] and addresses 149 them via specific modules in treatment [48]. In this sense, a modular-based psychotherapy 150 provides a structured approach of tailoring treatments to fit patient needs by allowing greater 151 flexibility to consider interindividual differences and comorbidity $[49,50]$. The modules, as sets 152 of independent but combinable functional units, focus on common transdiagnostic dysfunctions 153 and offer skills to improve e.g. emotion regulation, social competence, empathy, or selfmotivation. There is only one study [51] in which emotion regulation skills were successfully added to CBT in depressed patients that had sufficient statistical power to detect a clinically significant effect.

\section{Modular-Based Psychotherapy (MoBa)}

158 Empirical support for the effectiveness of modular approaches following decision flowcharts is emerging lately [50,52]. For instance, Weisz and colleagues [49] conducted a large randomized controlled trial $(\mathrm{RCT})$ in which a Modular Approach to Therapy for Children with

161 Anxiety, Depression, Trauma, or Conduct Problems (MATCH) outperformed standard manual

162 treatment as well as care as usual (CAU). The superiority of MATCH was found to be sustained 163 in a two-year follow-up [53] and was replicated in a more recent trial [54]. Another example of 164 a modular approach to psychotherapy is Behavioural Interventions for Anxiety in Children with 
Autism (BIACA) [55]. By using a modular format and including an algorithm to guide the selection of modules, it offers a treatment approach for several anxiety disorders and obsessive-compulsive disorder for youths on the autism spectrum. BIACA was superior to waitlist and CAU in several RCTs (e.g. [56]). In adults, a still ongoing RCT [57] assesses the feasibility of a modular transdiagnostic intervention for mood, stressor-related and anxiety disorders (HARMONIC trial) in preparation for a later-stage trial. This represents early signs of a significant paradigm shift away from single-diagnosis approaches towards dimensional, transdiagnostic, and modular-based conceptualizations [58,46].

The here proposed rationale for a modular-based psychotherapy ( $\mathrm{MoBa}$ ) for depressed patients with comorbidity and a history of $\mathrm{CM}$ is two-fold: First, to include patients regularly seen in clinical practice showing a) more often comorbid and heterogeneous complaints than the samples usually included in RCTs and b) a limited treatment response to standard disorderspecific approaches. Second, tailoring the treatment to the specific characteristics and needs of patients with $\mathrm{CM}$ and comorbid depression can ensure that the psychotherapeutic process is responsive and may reach better treatment results. The $\mathrm{MoBa}$ intervention aims at interpersonal and emotional maturation by overcoming social threat hypersensitivity and interpersonal avoidance patterns and improving poor mentalization as well as poor emotion regulation capacities. The rationale is supported by previous trials with empirically supported treatments such as CBASP for chronic depression [59-62], MBCT for depression prevention and treatment [63-66], and Mentalization-Based Therapy (MBT) [67] for borderline personality disorder $[68,69]$. In the here used design, MoBa complements standard CBT with modules compiling specific elements from CBASP, MBCT, and MBT focusing on three disturbed systems (Figure 1). Those systems are part of the RDoC model and have been shown to be critically related to $\mathrm{CM}$ :

I) the negative valence system (acute, potential, and sustained threat): social threat response and avoidance behaviour [34,9];

II) the system of social processes: perception and understanding of self and others (understanding mental states), social communication, attachment [70,37,38]; 
III) the arousal system: emotion awareness and arousal regulation [40,71-73].

194

195

196

197

198

199

200

201

202

203

204

205

206

207

208

209

210

211

212

213

214

\section{[FIGURE 1]}

\section{Objectives}

This pilot study has a number of objectives appropriate to its status as a feasibility study:

1. Providing initial evidence for the efficacy of $\mathrm{MoBa}$ (reduction of clinician-rated depressive symptoms) as well as generating pilot data for the power calculation in terms of effect and sample size for a subsequent multicentre confirmatory trial.

2. Investigating the planned study design regarding the feasibility of recruitment, feasibility of applying cut-off values of self-reported deficits to select the modules, acceptability of the program to therapists and patients as well as patient ratings of 'usefulness' (both overall and in terms of individual modules). A crucial goal is to refine the algorithm for the selection of modules based on questionnaires.

3. Explore potential moderators of the primary outcome (in a hypothesis-generating exercise and to help refine the intervention).

\section{Methods and analysis}

\section{Study design}

The bicentric study will be conducted at the Department of Psychiatry and Psychotherapy, University Medical Center Freiburg, Germany, and the Department of General Psychiatry, University Medical Center Heidelberg, Germany. It is a parallel-arm RCT ( $\mathrm{N}=70)$ comparing MoBa with $\mathrm{CBT}$ in 20 individual sessions over 16 weeks of treatment (twice weekly in weeks 1-4, then once per week in weeks 5-16). Participants will be assessed for screening and at baseline, post-treatment and follow-up (six months after end of treatment).

\section{Study population and recruitment}

Seventy outpatients with episodic or persistent major depression, comorbidity and childhood maltreatment will be recruited. Key inclusion and exclusion criteria are:

Inclusion criteria: 
1. Age eligibility: $18-65$ years.

2. Episodic or persistent MDD or MDD superimposed on Dysthymia ("Double Depression") as the primary diagnosis (according to the SCID-5) [74].

3. A score of $>18$ on the Hamilton Rating Scale for Depression (HRSD-24) [75].

4. History of CM: at least moderate to severe in one or more of the five CTQ-categories (emotional neglect, emotional abuse, physical neglect, physical abuse, sexual abuse) [15].

5. Any psychiatric comorbidity according to the SCID-5 except for those described in the exclusion criteria below.

6. Exceeding the 'cut-off' value of at least one of the following measures (module questionnaires): 1) Rejection Sensitivity Questionnaire (RSQ, [76]) $\geq 9.88,2$ ) Interpersonal Reactivity Index (IRI, [77]) < 45, or 3) Difficulties in Emotion Regulation Scale-16 (DERS-16, [78]) $\geq 55.73$.

7. Written informed consent.

\section{Exclusion criteria:}

1. Acute risk of suicide.

2. Other current psychiatric disorders as primary diagnosis.

3. Comorbid schizophrenia, bipolar I disorder, organic disorder or substance dependence fulfilling criteria within the last 6 months.

4. Antisocial or borderline personality disorder (BPD). For BPD, up to three traits are allowed.

5. Severe cognitive impairment.

6. Serious medical condition (interfering with participation in regular sessions).

7. Other ongoing psychotherapy or psychotropic medication except antidepressant (e.g. selective serotonin reuptake inhibitor (SSRI) / serotonin-norepinephrine reuptake inhibitor (SNRI)) and/or sleep-inducing treatment at baseline if stable for at least three weeks before inclusion (four weeks for fluoxetine). Rescue medication is benzodiazepine for a maximum of 2 weeks or on-demand. 
247 Patients will be recruited through psychiatric and psychotherapeutic outpatient clinics and 248 private practices by announcement of the psychotherapy treatment offers. Approximately 120 249 patients will be pre-screened for eligibility by research assistants via telephone with a brief pre250 screening guide that has been successfully used in prior depression studies. A total of $N=70$ patients will be randomised (Figure 2).

\section{Sample size}

254 Due to the exploratory nature of the design and the lack of comparable studies, no formal 255 sample size calculation is possible. One of the major aims of this trial is to generate pilot data for a subsequent sample size calculation for a confirmatory study. With reference to Billingham et al. [79] a medium sample size of 30 patients per group in pilot trials seems to be reasonable for the generation of pilot data for such estimation. That results in a total of 60 patients. Noncompliance and/or dropout of patients after randomization are assumed to be at most $14 \%$. Therefore, 70 patients have to be randomized to observe the desired number of compliant patients, split in two groups for each of the two participating centres ( $F R=35, H D=35$; Figure 2).

\section{Outcomes}

The primary endpoint is the HRSD-24measured by blind, independent raters at the conclusion of the 16-week treatment period. All secondary endpoints are describe in Table 1.

\section{[TABLE 1]}

267 A comprehensive overview about the frequency and scope of all trial visits including all 268 assessments and measures is depicted below (figure 3).

\section{[FIGURE 3]}

\section{Adherence}

271 Study psychotherapists are in a completed or far advanced stage of psychotherapy training.

272 All therapist will execute CBT as well as MoBa interventions after thorough training to ensure 273 a high treatment quality (1.5-day training course in CBT, 2.5-day training course in MoBa). All 
274 trainings are led by clinical experts in the field. The training process for therapists includes the 275 supervision of one pilot case in each arm and an adherence rating for study certification. To 276 check for adherence in the further process and to support the supervision, a "Therapeutic 277 Element Checklist" is filled out by the therapists immediately after each session. Supervisors 278 will review the "Therapeutic Element Checklist" regularly in ongoing supervision. All therapy 279 sessions will be videotaped for adherence and supervision. Every 5th session will be 280 supervised by the responsible supervisor in biweekly video conference meetings and/or by 281 written feedback. Two clinical experts will conduct the diagnostic training of raters in SCID-5, 282 HRSD-24 and SOFAS and interrater reliability will be ensured.

\section{Experimental intervention: Modular-Based Psychotherapy}

The MoBa model complements standard CBT for depression with modules aiming at socioemotional cognitive deficits and compiling specific strategies from CBASP, MBT and mindfulness (figure 1). Content and implementation of the three modules are illustrated in Table 2.

[TABLE 2].

\section{Selection of modules}

291 The application of the modular intervention is preceded by a structured diagnostic assessment 292 of the patient's impaired systems (negative valence system, system of social processes, or 293 arousal system) according to the scores on the 1) Rejection Sensitivity Questionnaire (RSQ; 294 social threat response);2) Interpersonal Reactivity Index (IRI; mentalization, empathy), and 3) 295 Brief Version of the Difficulties in Emotion Regulation Scale (DERS-16; emotion awareness 296 and regulation). The corresponding modular interventions will be applied if the cut-off value in 297 one or more of these measures is exceeded. Since no a priori values are established, the cut298 offs used here are defined as one standard deviation above the general population mean, i.e. 299 the upper $16 \%[81,78]$. The problem(s) thus identified is/are assigned as the target for one, 
two or three of the modules (figure 3) according to the systematic treatment algorithm (figure $3014)$.

\section{[FIGURE 4]}

\section{Application of modules (time distribution)}

304 The modules are not simply added as separate components, but rather integrated into the 305 therapeutic process and course as add-on to the standard CBT procedure. Consequently, the 306 amount of time spent with single CBT-techniques (e.g. cognitive restructuring) will be reduced 307 with increasing number of modules and the procedure will be condensed to behavioural 308 activation (e.g. identifying and promoting pleasant activities) as the most effective component 309 of CBT [98]. Depending on the selected number of modules, approximately one third of the 310 time will be spent with basic CBT procedures and two thirds of the time with the application of 311 modules. Therapists will document the time, which is spent with CBT procedures or with single 312 modules, after each session.

\section{Control intervention: $C B T$}

314 CBT will be delivered according to the German standard manual by Hautzinger [99]. The main 315 CBT elements are 1. establishing therapeutic relationship, 2. psychoeducation, 3. behaviour 316 activation, 4. cognitive restructuring, and 5. maintenance and relapse prevention. CBT has 317 been shown to be efficacious in depressed patients in prior clinical trials [100,101], but not 318 specifically in this subgroup of depressed and comorbid patients exposed to CM.

\section{Randomisation}

320 The randomisation code will be generated by the Clinical Trials Unit Freiburg (CTU) using the 321 following procedure to ensure that treatment assignment is unbiased and concealed from 322 patients and investigator staff. Randomisation will be performed, stratified by site, in blocks of 323 variable length in a ratio of 1:1. The block lengths will be documented separately and will not 324 be disclosed to the sites. The randomisation code will be produced by validated programs based on the Statistical Analysis System (SAS). This dataset is included in Redcap so that patients can be randomised directly in the eCRF. 


\section{Blinding}

All clinical ratings will be completed by trained and independent raters blinded to treatment assignment. Each of the sites implements procedures to mask a patient treatment assignment from the person who will evaluate the results of the clinical ratings through the following: 1) locating the raters at a separate physical location, and 2) reminding the patients at each visit not to mention anything that might reveal their treatment condition to the independent evaluator.

\section{Data Management and Monitoring}

Study data will be entered in pseudonymised form in a study database by authorized and trained members of the study team via electronic case report forms (eCRF). The data management will be performed with REDCap ${ }^{\mathrm{TM}}$ Version 9, a fully web based remote data entry system based on web forms, which is developed and maintained by the REDCap Consortium (redcap@vanderbilt.edu). This system uses built-in security features to prevent unauthorized access to patient data, including an encrypted transport protocol for data transmission from the participating sites to the study database. An audit trail provides a history of the data entered, changed, or deleted, indicating the processor and date. Monitoring is performed by CTU. Risk-based monitoring will be done according to ICH-GCP E6 (R2) and standard operating procedures (SOP) to ensure patient`s safety and integrity of clinical trial data.

\section{Statistical Analysis}

Before the start of the final analysis, a detailed statistical analysis plan will be prepared. This will be completed during the 'blind review' of the data, at the latest. The primary efficacy analysis will be performed according to the intention-to-treat (ITT) principle and will therefore be based on the full analysis set including all randomized patients. Patients are analysed as randomised regardless of any protocol deviations. This analysis corresponds to the analysis of the treatment policy estimand. The effects of CBT and MoBa with respect to the HRSD-24 score after 16 weeks of treatment (primary endpoint) will be estimated within a linear regression model, and the two-sided 95\% confidence interval will be calculated for the 
354 treatment effect. The model will include treatment and study centre as independent variables, 355 as well as baseline HRSD-24 score. A conservative assumption of the effect size anticipated 356 for the subsequent confirmative trial will be derived from these analyses by a combination of 357 clinical and statistical judgement. Secondary endpoints will be analysed descriptively in a similar fashion as the primary outcome, using regression models as appropriate for the respective type of data with two-sided $95 \%$ confidence intervals. All secondary analyses are exploratory and are interpreted in a descriptive fashion.

361 The safety analysis includes calculation and comparison of frequencies and rates of serious adverse events. Furthermore, statistical methods are used to assess the quality of data and the homogeneity of intervention groups. Data should be collected regardless of the patients' adherence to the protocol, especially on the clinical outcome, to obtain the best approximation to the full analysis set. Data should also be collected on other therapies received post dropout. Patients with missing follow-up will be excluded. As the only available measurement of the patient is taken at baseline and the primary aim is feasibility, this can be considered as an adequate strategy. The reasons for missing post baseline values will be collected and will be taken into consideration for the subsequent confirmatory trial.

Study results will be reported according to CONSORT guidelines. Further details of the statistical analysis will be fixed before data base lock and start of the analysis. The responsible

372 biostatistician will remain blind for treatment allocation throughout the study. For further 373 information regarding the statistical analysis, see the extensive study protocol publicly

\section{4 accessible}

at

375 https://www.drks.de/drks web/navigate.do?navigationld=trial.HTML\&TRIAL ID=DRKS00022

$376 \quad \underline{093 .}$

\section{Ethics and dissemination:}

378 This study obtained approval from the independent Ethics Committees of the University of 379 Freiburg in August 2020 and the University of Heidelberg in October 2020. Additionally, the 380 administrative department for governance and quality of the University Medical Center 
381 Freiburg verified GCP conformity. All findings will be disseminated broadly via peer-reviewed 382 articles in scientific journals and contributions to national and international conferences.

383

384

385

386

387

388

389

390

391

392

393

394

395

396

397

398

399

400

401

402

403

404

405

406

407

\section{Consent to participate}

At first contact, all prospects will be informed about the study in detail and will receive standardized participant information sheets. At screening, voluntary written informed consent for study participation and storage, evaluation and transfer of study-related data will be obtained from each study participant by research associates of the respective study centre. Withdrawal of written consent is possible at any time, without giving reasons. In the event of a withdrawal of the informed consent, patients can decide whether their data should be deleted or destroyed or whether they can be used in anonymised form for this research project.

\section{Safety/harms}

Side effects of evidence- based psychotherapies are fortunately rather rare (e.g. [102,103]). According to the most recent meta-analysis, only approximately $5 \%$ of patients deteriorate while in psychotherapeutic treatment [3]. Adverse Events (AE; e.g. private/occupational stress or conflicts in the patient-therapist relationship) and Serious Adverse Events (SAE; e.g. severe events requiring stationary medical treatment or with potential permanent damage) are screened for at every assessment or therapy session. AEs have to be reported to the principal investigators (ES, SH) and SAEs to the independent experts. In addition, on-site data monitoring will be regularly conducted by a clinical monitor from CTU to ensure patients' safety and integrity of the clinical data in adherence to the study protocol, as well as to check data quality and accuracy. Individual trial participation will be stopped if one of the following discontinuation criteria occurs:

a) Active suicidality

b) The physical health of the patient is at risk according to clinical judgment

c) Occurrence of an AE/SAE with therapeutic implications incompatible with the study

d) Newly occurring exclusion criteria (demanding further procedures not compatible with the continuation of the study participation) 
e) Withdrawal of the informed consent

409

If the study principal investigator or the co-principal investigator have serious ethical concerns because of the performance at one of the sites or severe safety concerns become apparent to the independent experts, the whole trial will be discontinued.

\section{Trial Status}

Official study begin was in May 2020. The first patient was included in December 2020. Within the first months of recruiting, there were no difficulties regarding the recruitment and inclusion of eligible patients, or the implementation of the MoBa and CBT treatments. Due to the ongoing COVID-19 pandemic, all in person contacts (assessments as well as psychotherapy sessions) are done while wearing appropriate face masks (surgical or FFP2) according to the national guidelines and the respective guidelines of the University Medical Centers in Freiburg and Heidelberg. The end of treatment is expected for July 2022 and data collection aims to be completed in March 2023.

\section{Discussion}

Most evidence-based treatment protocols are single-disorder-specific manuals disregarding common comorbidities and transdiagnostic clinical phenomena as sequelae of early trauma and childhood adversities. This leaves a mismatch between the available disorder-specific manuals and the clinical reality. Many clinicians consider the use of evidence-based manuals as challenging or even inadequate for their daily work and report resistances to the 'oversimplified', 'rigid', 'inflexible' or 'flawed' rationales and the 'extensive efforts' needed to maintain up-to-date knowledge by ongoing training [104]. Even attending evidence-based workshops has little impact on clinicians' decisions to use evidence-based treatment protocols in their practice resulting in the well-known underutilization in community settings $[105,106]$. In contrast to conventional evidence-based treatment protocols, a modular-based psychotherapy supports the eclectic approach of most clinicians by providing them with an evidence-based treatment algorithm to combine and integrate available treatment modules as independent but combinable sets of functional units systematically. This reduces the perceived challenges of 
435 using evidence-based approaches by ensuring a high flexibility and goodness-of-fit within a 436 systematic framework for personalised treatments. By optimally tailoring module selection and 437 application to the specific needs of each patient, MoBa has great potential to improve the 438 currently unsatisfying results of psychotherapeutic treatments in research and clinical practice 439 as a bridge between disorder-specific and personalised approaches.

\section{Declarations}

\section{Availability of data and materials}

The datasets used and/or analysed during the current study are available from the corresponding author on reasonable request.

\section{Patient and public involvement}

Neither patients nor public were systematically involved in designing this study. Feedback is constantly collected from participants on their experience of participating and implemented in conducting this trial. The main results will be disseminated to trial participants and systematic patient and public involvement in the development of a subsequent multicentre confirmatory trial will be implemented.

\section{Competing interests}

451 The authors declare the following competing interests: ME received minor book royalties. SH received minor royalties for books with chapters on modular psychotherapy. HP and CJ declare no competing interests. $\mathrm{MH}$ received book royalties from several publishers. ES received book royalties and honoraria for workshops and presentations relating to Interpersonal Psychotherapy and CBASP.

\section{$456 \quad$ Funding}

457 The clinical trial is financially supported by the German Research Foundation (Deutsche 458 Forschungsgemeinschaft, DFG; GZ: SCHR 443/16-1). The funders do not control the final 459 decision regarding any of aspects of the trial: design, conduct, data analysis and interpretation, manuscript writing, and dissemination of trial results. 


\section{Authors contributions}

462

$\mathrm{ME}, \mathrm{ES}$ and $\mathrm{SH}$ were the main contributors in drafting this manuscript. ES and $\mathrm{SH}$ were the main contributors in designing this study with support by ME. CJ provided expertise on data monitoring, data management and statistical analyses. All authors provided feedback on the initial draft of the manuscript and read and approved the final version.

\section{Acknowledgements}

We would like to thank Prof. Fritz Hohagen, Prof. Klaus Lieb and Prof. Matthias Backenstraß for their contributions as independent experts, as well as Dr. Thomas Fangmeier for his valuable assistance in determining the module cut-offs for the initial grant. Furthermore, we are grateful for the participation of Dr. Anne Külz and Prof. Svenja Taubner for their ongoing supervision and therapist trainings as experts of their fields in MBCT and MBT.

\section{References}

1. Beck AT. Cognitive Therapy of Depression. Guilford Press; 1979.

2. Klerman GL, Weissman MM, Rounsaville BJ, Chevron E. Interpersonal Psychotherapy of Depression. Basic Books; 1984.

3. Cuijpers P, Karyotaki E, Ciharova M, Miguel C, Noma H, Furukawa TA. The effects of psychotherapies for depression on response, remission, reliable change, and deterioration: A meta-analysis. Acta Psychiatrica Scandinavica. 2021. doi:10.1111/acps. 13335

4. Berlim MT, Turecki G. What is the meaning of treatment resistant/refractory major depression (TRD)? A systematic review of current randomized trials. European Neuropsychopharmacology. 2007;17(11):696-707. doi:10.1016/j.euroneuro.2007.03.009

5. Holtzheimer PE, Mayberg HS. Stuck in a rut: Rethinking depression and its treatment. Trends in Neurosciences. 2011;34(1):1-9. doi:10.1016/j.tins.2010.10.004

6. Kessler RC, Berglund P, Demler O, Jin R, Merikangas KR, Walters EE. Lifetime Prevalence and Age-of-Onset Distributions of DSM-IV Disorders in the National Comorbidity Survey Replication. Archives of General Psychiatry. 2005;62(6):593-602. doi:10.1001/archpsyc.62.6.593

7. Lamers F, Oppen P van, Comijs HC, et al. Comorbidity Patterns of Anxiety and Depressive Disorders in a Large Cohort Study: The Netherlands Study of Depression and Anxiety (NESDA). J Clin Psychiatry. 2011;72(3):0-0. doi:10.4088/JCP.10m06176blu

8. Roca M, Gili M, Garcia-Garcia M, et al. Prevalence and comorbidity of common mental disorders in primary care. Journal of Affective Disorders. 2009;119(1):52-58. doi:10.1016/j.jad.2009.03.014

9. Klein JP, Roniger A, Schweiger U, Späth C, Brodbeck J. The Association of Childhood Trauma and Personality Disorders With Chronic Depression: A Cross-Sectional Study in Depressed Outpatients. J Clin Psychiatry. 2015;76(6):0-0. doi:10.4088/JCP.14m09158

10. Papakostas GI, Fava M. Predictors, moderators, and mediators (correlates) of treatment outcome in major depressive disorder. Dialogues Clin Neurosci. 2008;10(4):439-451. doi:10.31887/DCNS.2008.10.4/gipapakostas

11. Souery D, Oswald P, Massat I, et al. Clinical Factors Associated With Treatment Resistance in Major Depressive Disorder: Results From a European Multicenter Study. J Clin Psychiatry. 2007;68(7):0-0.

12. Goddard E, Wingrove J, Moran P. The impact of comorbid personality difficulties on response to IAPT treatment for depression and anxiety. Behavior Research and Therapy. 2015;73:1-7. doi:10.1016/j.brat.2015.07.006 
13. Agosti V. Predictors of remission from chronic depression: A prospective study in a nationally representative sample. Comprehensive Psychiatry. 2014;55(3):463-467. doi:10.1016/j.comppsych.2013.09.016

14. Frank E, Cassano GB, Rucci $P$, et al. Predictors and moderators of time to remission of major depression with interpersonal psychotherapy and SSRI pharmacotherapy. Psychological Medicine. 2011;41(1):151-162. doi:10.1017/S0033291710000553

15. Bernstein DP, Stein JA, Newcomb MD, et al. Development and validation of a brief screening version of the Childhood Trauma Questionnaire. Child Abuse \& Neglect. 2003;27(2):169-190. doi:10.1016/S0145-2134(02)00541-0

16. Nelson J, Klumparendt A, Doebler P, Ehring T. Childhood maltreatment and characteristics of adult depression: Meta-analysis. The British Journal of Psychiatry. 2017;210(2):96-104. doi:10.1192/bjp.bp. 115.180752

17. Struck N, Krug A, Yuksel D, et al. Childhood maltreatment and adult mental disorders - the prevalence of different types of maltreatment and associations with age of onset and severity of symptoms. Psychiatry Research. 2020;293:113398. doi:10.1016/j.psychres.2020.113398

18. Wiersma JE, Hovens JGFM, Oppen P van, Giltay EJ, Schaik DJF van, Penninx BWJH. The Importance of Childhood Trauma and Childhood Life Events for Chronicity of Depression in Adults. J Clin Psychiatry. 2009;70(7):0-0. doi:10.4088/JCP.08m04521

19. Humphreys KL, LeMoult J, Wear JG, Piersiak HA, Lee A, Gotlib IH. Child maltreatment and depression: A meta-analysis of studies using the Childhood Trauma Questionnaire. Child Abuse \& Neglect. 2020;102:104361. doi:10.1016/j.chiabu.2020.104361

20. Infurna MR, Reichl C, Parzer P, Schimmenti A, Bifulco A, Kaess M. Associations between depression and specific childhood experiences of abuse and neglect: A meta-analysis. Journal of Affective Disorders. 2016;190:47-55. doi:10.1016/j.jad.2015.09.006

21. Mandelli L, Petrelli C, Serretti A. The role of specific early trauma in adult depression: A metaanalysis of published literature. Childhood trauma and adult depression. European Psychiatry. 2015;30(6):665-680. doi:10.1016/i.eurpsy.2015.04.007

22. Bausch $\mathrm{P}$, Fangmeier T, Meister R, et al. The Impact of Childhood Maltreatment on Long-Term Outcomes in Disorder-Specific vs. Nonspecific Psychotherapy for Chronic Depression. Journal of Affective Disorders. 2020;272:152-157. doi:10.1016/j.jad.2020.03.164

23. Teicher MH, Samson JA. Childhood maltreatment and psychopathology: A case for ecophenotypic variants as clinically and neurobiologically distinct subtypes. Am J Psychiatry. 2013;170(10):1114-1133. doi:10.1176/appi.ajp.2013.12070957

24. Shirk SR, DePrince AP, Crisostomo PS, Labus J. Cognitive behavioral therapy for depressed adolescents exposed to interpersonal trauma: An initial effectiveness trial. Psychotherapy. 2014;51(1):167-179. doi:10.1037/a0034845

25. Williams JMG, Crane C, Barnhofer T, et al. Mindfulness-based cognitive therapy for preventing relapse in recurrent depression: A randomized dismantling trial. Journal of Consulting and Clinical Psychology. 2014;82(2):275-286. doi:10.1037/a0035036

26. Nemeroff CB, Heim CM, Thase ME, et al. Differential Responses to Psychotherapy Versus Pharmacotherapy in Patients With Chronic Forms of Major Depression and Childhood Trauma. FOC. 2005;3(1):131-135. doi:10.1176/foc.3.1.131

27. Klein JP, Erkens N, Schweiger U, et al. Does Childhood Maltreatment Moderate the Effect of the Cognitive Behavioral Analysis System of Psychotherapy versus Supportive Psychotherapy in Persistent Depressive Disorder? PPS. 2018;87(1):46-48. doi:10.1159/000484412

28. McCullough JP. Treatment for Chronic Depression. Cognitive Behavioral Analysis System of Psychotherapy. Guilford Press; 2000.

29. McCullough J, Schramm E, Penberthy JK. CBASP as a Distinctive Treatment for Persistent Depressive Disorder: Distinctive Features. Routledge; 2015.

30. Bertsch K, Krauch M, Stopfer K, Haeussler K, Herpertz SC, Gamer M. Interpersonal Threat Sensitivity in Borderline Personality Disorder: An Eye-Tracking Study. Journal of Personality Disorders. 2017;31(5):647-670. doi:10.1521/pedi $2017 \quad 31273$

31. Chu DA, Bryant RA, Gatt JM, Harris AWF. Failure to differentiate between threat-related and positive emotion cues in healthy adults with childhood interpersonal or adult trauma. Journal of Psychiatric Research. 2016;78:31-41. doi:10.1016/i.jpsychires.2016.03.006

32. Herpertz SC, Bertsch K. The social-cognitive basis of personality disorders. Current Opinion in Psychiatry. 2014;27(1):73-77. doi:10.1097/YCO.0000000000000026

33. Bertsch K, Gamer M, Schmidt B, et al. Oxytocin and Reduction of Social Threat Hypersensitivity in Women With Borderline Personality Disorder. AJP. 2013;170(10):1169-1177. doi:10.1176/appi.ajp.2013.13020263 
34. Shapero BG, Black SK, Liu RT, et al. Stressful Life Events and Depression Symptoms: The Effect of Childhood Emotional Abuse on Stress Reactivity. Journal of Clinical Psychology. 2014;70(3):209-223. doi:10.1002/jclp.22011

35. Erhardt A, Spoormaker VI. Translational Approaches to Anxiety: Focus on Genetics, Fear Extinction and Brain Imaging. Curr Psychiatry Rep. 2013;15(12):417. doi:10.1007/s11920-0130417-9

36. Schnell K, Herpertz SC. Emotion Regulation and Social Cognition as Functional Targets of Mechanism-Based Psychotherapy in Major Depression With Comorbid Personality Pathology. Journal of Personality Disorders. 2018;32(Supplement):12-35. doi:10.1521/pedi.2018.32.supp.12

37. Schnell K, Bluschke S, Konradt B, Walter H. Functional relations of empathy and mentalizing: An fMRI study on the neural basis of cognitive empathy. Neurolmage. 2011;54(2):1743-1754. doi:10.1016/j. neuroimage.2010.08.024

38. Mattern $\mathrm{M}$, Walter $\mathrm{H}$, Hentze $\mathrm{C}$, et al. Behavioral Evidence for an Impairment of Affective Theory of Mind Capabilities in Chronic Depression. PSP. 2015;48(4):240-250. doi:10.1159/000430450

39. Weissman DG, Bitran D, Miller AB, Schaefer JD, Sheridan MA, McLaughlin KA. Difficulties with emotion regulation as a transdiagnostic mechanism linking child maltreatment with the emergence of psychopathology. Development and Psychopathology. 2019;31(3):899-915. doi:10.1017/S0954579419000348

40. Cloitre M, Stovall-McClough C, Zorbas $P$, Charuvastra A. Attachment organization, emotion regulation, and expectations of support in a clinical sample of women with childhood abuse histories. Journal of Traumatic Stress. 2008;21(3):282-289. doi:10.1002/its.20339

41. Lippard ETC, Nemeroff CB. The Devastating Clinical Consequences of Child Abuse and Neglect: Increased Disease Vulnerability and Poor Treatment Response in Mood Disorders. AJP. 2020;177(1):20-36. doi:10.1176/appi.ajp.2019.19010020

42. Bock J, Wainstock T, Braun K, Segal M. Stress In Utero: Prenatal Programming of Brain Plasticity and Cognition. Biological Psychiatry. 2015;78(5):315-326. doi:10.1016/i.biopsych.2015.02.036

43. Fonagy P, Luyten P. Fidelity vs. flexibility in the implementation of psychotherapies: Time to move on. World Psychiatry. 2019;18(3):270-271. doi:10.1002/wps.20657

44. Barlow DH, Bullis JR, Comer JS, Ametaj AA. Evidence-Based Psychological Treatments: An Update and a Way Forward. Annual Review of Clinical Psychology. 2013;9(1):1-27. doi:10.1146/annurev-clinpsy-050212-185629

45. Lyon AR, Lau AS, McCauley E, Vander Stoep A, Chorpita BF. A case for modular design: Implications for implementing evidence-based interventions with culturally diverse youth. Professional Psychology: Research and Practice. 2014;45(1):57-66. doi:10.1037/a0035301

46. Insel TR, Cuthbert BN. Brain disorders? Precisely. Science. 2015;348(6234):499-500. doi:10.1126/science.aab2358

47. Harvey AG, Watkins E, Mansell W. Cognitive Behavioral Processes Across Psychological Disorders: A Transdiagnostic Approach to Research and Treatment. Oxford University Press; 2004.

48. Barlow DH, Allen LB, Choate ML. Toward a unified treatment for emotional disorders. Behavior Therapy. 2004;35(2):205-230. doi:10.1016/S0005-7894(04)80036-4

49. Weisz JR, Chorpita BF, Palinkas LA, et al. Testing Standard and Modular Designs for Psychotherapy Treating Depression, Anxiety, and Conduct Problems in Youth: A Randomized Effectiveness Trial. Archives of General Psychiatry. 2012;69(3):274-282. doi:10.1001/archgenpsychiatry.2011.147

50. Ng MY, Weisz JR. Annual Research Review: Building a science of personalised intervention for youth mental health. Journal of Child Psychology and Psychiatry. 2016;57(3):216-236. doi:10.1111/jcpp. 12470

51. Berking M, Ebert D, Cuijpers P, Hofmann SG. Emotion Regulation Skills Training Enhances the Efficacy of Inpatient Cognitive Behavioral Therapy for Major Depressive Disorder: A Randomized Controlled Trial. PPS. 2013;82(4):234-245. doi:10.1159/000348448

52. Cuijpers P, Ebert DD, Acarturk C, Andersson G, Cristea IA. Personalised Psychotherapy for Adult Depression: A Meta-Analytic Review. Behavior Therapy. 2016;47(6):966-980. doi:10.1016/i.beth.2016.04.007

53. Chorpita BF, Weisz JR, Daleiden EL, et al. Long-term outcomes for the Child STEPs randomized effectiveness trial: A comparison of modular and standard treatment designs with usual care. Journal of Consulting and Clinical Psychology. 2013;81(6):999-1009. doi:10.1037/a0034200

54. Chorpita BF, Daleiden EL, Park AL, et al. Child STEPs in California: A cluster randomized effectiveness trial comparing modular treatment with community implemented treatment for youth with anxiety, depression, conduct problems, or traumatic stress. Journal of Consulting and Clinical Psychology. 2017;85(1):13-25. doi:10.1037/ccp0000133 
55. Wood JJ, Drahota A, Sze K, Har K, Chiu A, Langer DA. Cognitive behavioral therapy for anxiety in children with autism spectrum disorders: A randomized, controlled trial. Journal of Child Psychology and Psychiatry. 2009;50(3):224-234. doi:10.1111/j.1469-7610.2008.01948.x

56. Storch EA, Arnold EB, Lewin AB, et al. The Effect of Cognitive-Behavioral Therapy Versus Treatment as Usual for Anxiety in Children With Autism Spectrum Disorders: A Randomized, Controlled Trial. Journal of the American Academy of Child \& Adolescent Psychiatry. 2013;52(2):132-142.e2. doi:10.1016/j.jaac.2012.11.007

57. Black M, Hitchcock C, Bevan A, et al. The HARMONIC trial: Study protocol for a randomised controlled feasibility trial of Shaping Healthy Minds - a modular transdiagnostic intervention for mood, stressor-related and anxiety disorders in adults. BMJ Open. 2018;8(8):e024546. doi:10.1136/bmjopen-2018-024546

58. Dalgleish T, Black M, Johnston D, Bevan A. Transdiagnostic approaches to mental health problems: Current status and future directions. Journal of Consulting and Clinical Psychology. 20200217;88(3):179. doi:10.1037/ccp0000482

59. Negt $P$, Brakemeier E-L, Michalak J, Winter L, Bleich S, Kahl KG. The treatment of chronic depression with cognitive behavioral analysis system of psychotherapy: A systematic review and meta-analysis of randomized-controlled clinical trials. Brain and Behavior. 2016;6(8):e00486. doi:10.1002/brb3.486

60. Keller MB, McCullough JP, Klein DN, et al. A Comparison of Nefazodone, the Cognitive Behavioral-Analysis System of Psychotherapy, and Their Combination for the Treatment of Chronic Depression. New England Journal of Medicine. 2000;342(20):1462-1470. doi:10.1056/NEJM200005183422001

61. Schramm E, Kriston L, Zobel I, et al. Effect of Disorder-Specific vs Nonspecific Psychotherapy for Chronic Depression: A Randomized Clinical Trial. JAMA Psychiatry. 2017;74(3):233-242. doi:10.1001/jamapsychiatry. 2016.3880

62. Schramm E, Kriston L, Elsaesser M, et al. Two-Year Follow-Up after Treatment with the Cognitive Behavioral Analysis System of Psychotherapy versus Supportive Psychotherapy for Early-Onset Chronic Depression. PPS. 2019;88(3):154-164. doi:10.1159/000500189

63. McCartney M, Nevitt S, Lloyd A, Hill R, White R, Duarte R. Mindfulness-based cognitive therapy for prevention and time to depressive relapse: Systematic review and network meta-analysis. Acta Psychiatrica Scandinavica. 2021;143(1):6-21. doi:10.1111/acps.13242

64. Goldberg SB, Tucker RP, Greene PA, Davidson RJ, Kearney DJ, Simpson TL. Mindfulness -based cognitive therapy for the treatment of current depressive symptoms: A meta-analysis. Cognitive Behavior Therapy. 2019;48(6):445-462. doi:10.1080/16506073.2018.1556330

65. Piet $J$, Hougaard $E$. The effect of mindfulness-based cognitive therapy for prevention of relapse in recurrent major depressive disorder: A systematic review and meta-analysis. Clinical Psychology Review. 2011;31(6):1032-1040. doi:10.1016/j.cpr.2011.05.002

66. Kuyken W, Hayes R, Barrett B, et al. Effectiveness and cost-effectiveness of mindfulness-based cognitive therapy compared with maintenance antidepressant treatment in the prevention of depressive relapse or recurrence (PREVENT): A randomised controlled trial. The Lancet. 2015;386(9988):63-73. doi:10.1016/S0140-6736(14)62222-4

67. Bateman AW, Fonagy P. Handbook of Mentalizing in Mental Health Practice. American Psychiatric Publishing, Inc.; 2012.

68. Malda-Castillo J, Browne C, Perez-Algorta G. Mentalization-based treatment and its evidencebase status: A systematic literature review. Psychology and Psychotherapy: Theory, Research and Practice. 2019;92(4):465-498. doi:10.1111/papt.12195

69. Bateman A, Fonagy P. Randomized Controlled Trial of Outpatient Mentalization-Based Treatment Versus Structured Clinical Management for Borderline Personality Disorder. AJP. 2009;166(12):1355-1364. doi:10.1176/appi.ajp.2009.09040539

70. Zilberstein K. Neurocognitive considerations in the treatment of attachment and complex trauma in children. Clin Child Psychol Psychiatry. 2014;19(3):336-354. doi:10.1177/1359104513486998

71. Hofmann M, Fehlinger T, Stenzel N, Rief W. The Relationship Between Skill Deficits and Disability-A Transdiagnostic Study. Journal of Clinical Psychology. 2015;71(4):413-421. doi:10.1002/jclp.22156

72. Fehlinger T, Stumpenhorst M, Stenzel N, Rief W. Emotion regulation is the essential skill for improving depressive symptoms. Journal of Affective Disorders. 2013;144(1):116-122. doi:10.1016/i.jad.2012.06.015

73. Ochsner KN, Silvers JA, Buhle JT. Functional imaging studies of emotion regulation: A synthetic review and evolving model of the cognitive control of emotion. Ann N Y Acad Sci. 2012;1251:E1-24. doi:10.1111/j.1749-6632.2012.06751.x

74. Beesdo-Baum K, Zaudig M, Wittchen H-U. Strukturiertes Klinisches Interview Für DSM-5 [The Structured Clinical Interview for DSM-5-Clinician Version] (SCID-5-CV). Hogrefe; 2019. 
75. Hamilton M. Development of a Rating Scale for Primary Depressive Illness. British Journal of Social and Clinical Psychology. 1967;6(4):278-296. doi:10.1111/j.2044-8260.1967.tb00530.x

76. Downey G, Feldman SI. Implications of rejection sensitivity for intimate relationships. Journal of Personality and Social Psychology. 1996;70(6):1327-1343. doi:10.1037/0022-3514.70.6.1327

77. Davis $\mathrm{MH}$. Measuring individual differences in empathy: Evidence for a multidimensional approach. Journal of Personality and Social Psychology. 1983;44(1):113-126. doi:10.1037/0022-3514.44.1.113

78. Bjureberg J, Ljótsson B, Tull MT, et al. Development and Validation of a Brief Version of the Difficulties in Emotion Regulation Scale: The DERS-16. J Psychopathol Behav Assess. 2016;38(2):284-296. doi:10.1007/s10862-015-9514-X

79. Billingham SA, Whitehead AL, Julious SA. An audit of sample sizes for pilot and feasibility trials being undertaken in the United Kingdom registered in the United Kingdom Clinical Research Network database. BMC Med Res Methodol. 2013;13(1):104. doi:10.1186/1471-2288-13-104

80. Munder T, Wilmers F, Leonhart R, Linster HW, Barth J. Working Alliance Inventory-Short Revised (WAI-SR): Psychometric properties in outpatients and inpatients. Clinical Psychology \& Psychotherapy. 2010;17(3):231-239. doi:10.1002/cpp.658

81. Staebler K, Helbing E, Rosenbach C, Renneberg B. Rejection sensitivity and borderline personality disorder. Clinical Psychology \& Psychotherapy. 2011;18(4):275-283. doi:10.1002/cpp. 705

82. Paulus C. Der Saarbrücker Persönlichkeitsfragebogen zur Messung von Empathie (SPF-IRI). Published 2006. http://bildungswissenschaften.unisaarland. de/personal/paulus/hom epage/empathie.html

83. Hausberg MC, Schulz H, Piegler T, et al. Is a self-rated instrument appropriate to assess mentalization in patients with mental disorders? Development and first validation of the Mentalization Questionnaire (MZQ). Psychotherapy Research. 2012;22(6):699-709. doi:10.1080/10503307.2012.709325

84. Gratz KL, Roemer L. Multidimensional Assessment of Emotion Regulation and Dysregulation: Development, Factor Structure, and Initial Validation of the Difficulties in Emotion Regulation Scale. Journal of Psychopathology and Behavioral Assessment. 2004;26(1):41-54. doi:10.1023/B:JOBA.0000007455.08539.94

85. Goldman HH, Skodol AE, Lave TR. Revising Axis V for DSM-IV: A Review of Measures of Social Functioning. Published online September 1, 1992. Accessed July 27, 2021. https://papers.ssrn.com/abstract $=2143992$

86. WHOQOL Group. Development of the World Health Organization WHOQOL-BREF Quality of Life Assessment. Psychological Medicine. 1998;28(3):551-558. doi:10.1017/S0033291798006667

87. Beck AT, Steer RA, Brown GK. Manual for the Beck Depression Inventory-II. Psychological Corperation; 1996.

88. Beck AT, Steer RA. Beck Anxiety Inventory Manual. Psychological Corperation; 1993.

89. Ehrenthal JC, Dinger U, Lamla A, Funken B, Schauenburg H. Evaluation der deutschsprachigen Version des Bindungsfragebogens „Experiences in Close Relationships - Revised” (ECR-RD). Psychother Psychosom Med Psychol. 2009;59(6):215-223. doi:10.1055/s-2008-1067425

90. Price CJ, Thompson EA. Measuring Dimensions of Body Connection: Body Awareness and Bodily Dissociation. The Journal of Alternative and Complementary Medicine. 2007;13(9):945-953. doi:10.1089/acm.2007.0537

91. Kroenke K, Spitzer RL. The PHQ-9: A New Depression Diagnostic and Severity Measure. Psychiatric Annals. 2002;32(9):509-515. doi:10.3928/0048-5713-20020901-06

92. Kiesler DJ. The 1982 Interpersonal Circle: A taxonomy for complementarity in human transactions. Psychological Review. 1983;90(3):185-214. doi:10.1037/0033-295X.90.3.185

93. Hölzel BK, Lazar SW, Gard T, Schuman-Olivier Z, Vago DR, Ott U. How Does Mindfulness Meditation Work? Proposing Mechanisms of Action From a Conceptual and Neural Perspective. Perspect Psychol Sci. 2011;6(6):537-559. doi:10.1177/1745691611419671

94. Arch JJ, Craske MG. Mechanisms of mindfulness: Emotion regulation following a focused breathing induction. Behavior Research and Therapy. 2006;44(12):1849-1858. doi:10.1016/j.brat.2005.12.007

95. Geschwind N, Peeters F, Drukker M, van Os J, Wichers M. Mindfulness training increases momentary positive emotions and reward experience in adults vulnerable to depression: A randomized controlled trial. Journal of Consulting and Clinical Psychology. 2011;79(5):618628. doi: $10.1037 / \mathrm{a} 0024595$

96. Khoury B, Lecomte T, Fortin G, et al. Mindfulness-based therapy: A comprehensive metaanalysis. Clinical Psychology Review. 2013;33(6):763-771. doi:10.1016/j.cpr.2013.05.005 
97. Davidson RJ, Kabat-Zinn J, Schumacher J, et al. Alterations in Brain and Immune Function Produced by Mindfulness Meditation. Psychosomatic Medicine. 2003;65(4):564-570. doi:10.1097/01.PSY.0000077505.67574.E3

98. Dimidjian S, Hollon SD, Dobson KS, et al. Randomized trial of behavioral activation, cognitive therapy, and antidepressant medication in the acute treatment of adults with major depression. Journal of Consulting and Clinical Psychology. 2006;74(4):658-670. doi:10.1037/0022006X.74.4.658

99. Hautzinger M. Kognitive Verhaltenstherapie Bei Depressionen. 7. Auflage. Beltz Psychologie Verlags Union; 2013.

100. Cuijpers P. Four decades of outcome research on psychotherapies for adult depression: An overview of a series of meta-analyses. Canadian Psychology/Psychologie canadienne. 2017;58(1):7-19. doi:10.1037/cap0000096

101. Barth J, Munder T, Gerger H, et al. Comparative Efficacy of Seven Psychotherapeutic Interventions for Patients with Depression: A Network Meta-Analysis. FOC. 2016;14(2):229-243. doi:10.1176/appi.focus. 140201

102. Linden M, Strauß B. Risiken und Nebenwirkungen von Psychotherapie: Erfassung, Bewältigung, Risikovermeidung. 1st ed. MWV Medizinisch Wissenschaftliche Verlagsgesellschaft; 2012.

103. Hoffmann SO, Rudolf G, Strauß B. Unerwünschte und schädliche Wirkungen von Psychotherapie. Psychotherapeut. 2008;53(1):4-16. doi:10.1007/s00278-007-0578-2

104. Cook SC, Schwartz AC, Kaslow NJ. Evidence-Based Psychotherapy: Advantages and Challenges. Neurotherapeutics. 2017;14(3):537-545. doi:10.1007/s13311-017-0549-4

105. Herschell AD, Kolko DJ, Baumann BL, Davis AC. The role of therapist training in the implementation of psychosocial treatments: A review and critique with recommendations. Clinical Psychology Review. 2010;30(4):448-466. doi:10.1016/i.cpr.2010.02.005

106. Ecker AH, O'Leary $\mathrm{K}$, Fletcher TL, et al. Training and supporting mental health providers to implement evidence-based psychotherapies in frontline practice. Translational Behavioral Medicine. 2021;(ibab084). doi:10.1093/tbm/ibab084

\section{Figure and Table Legends}

Figure 1: Overview of the targeted RDoC domains and their corresponding objectives, assessments and modules. A detailed description of the modules is given below.

Figure 2: Trial design and flow of patients.

Table 1: Primary and Secondary Endpoints and corresponding measures.

Figure 3. Frequency and scope of trial visits.

Figure 4: Decision Tree Algorithm for Modular-Based Psychotherapy.

Table 2: Content and implementation of modules. 
Figure 1: Overview of the targeted RDoC domains and their corresponding objectives, assessments and modules. A detailed description of the modules is given below.

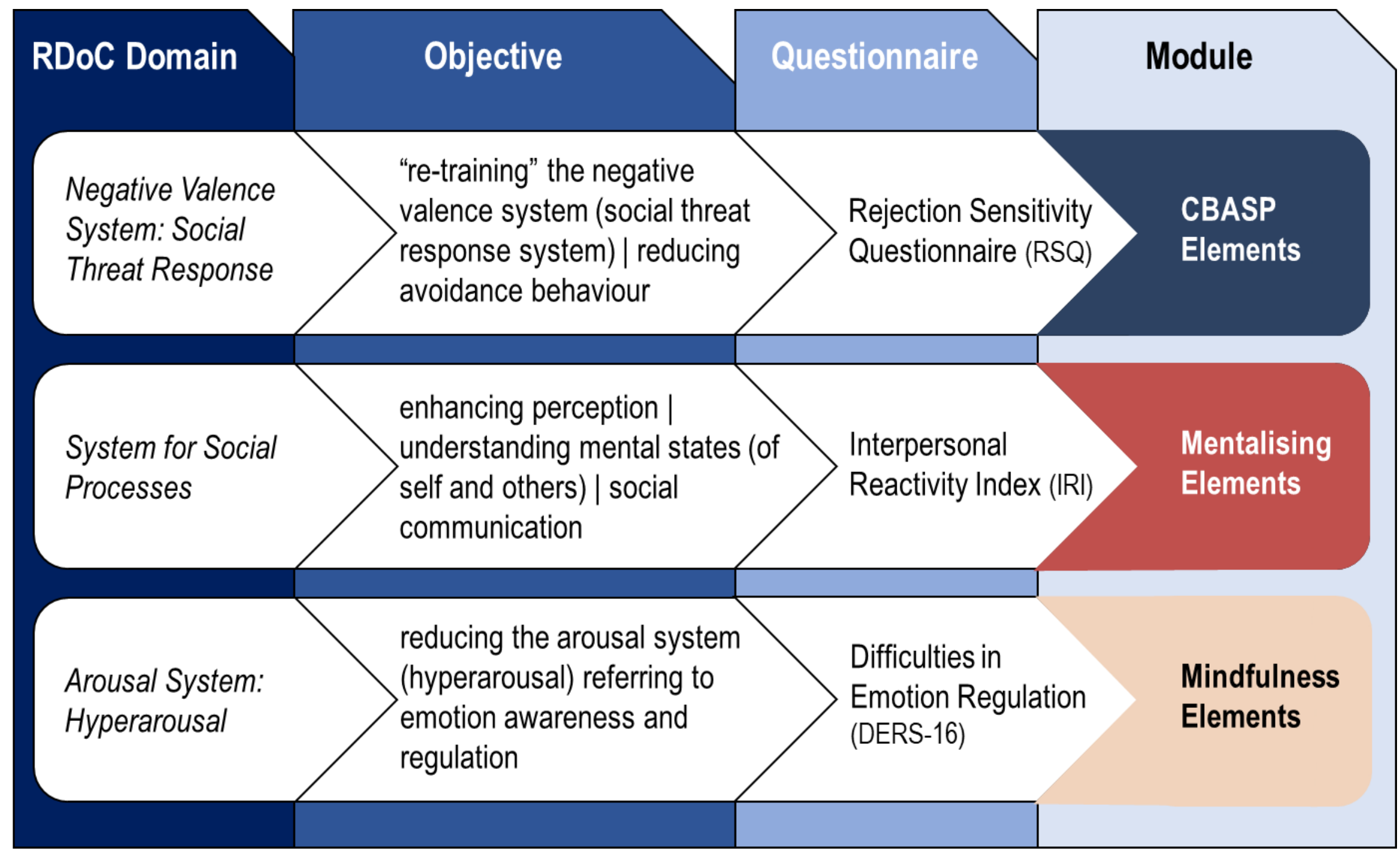


Figure 2: Trial design and flow of patients.

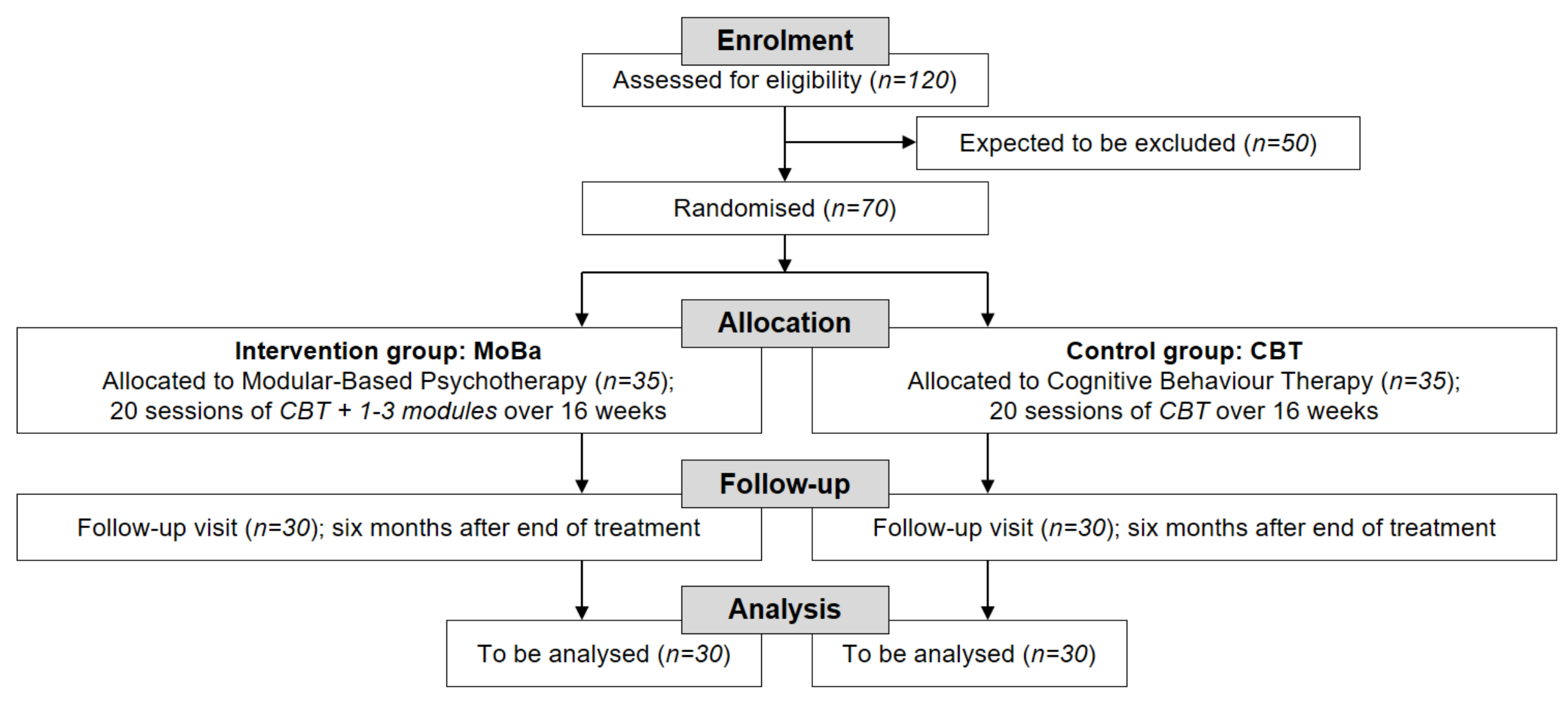


Figure 3. Frequency and scope of trial visits.

\begin{tabular}{|c|c|c|c|c|c|c|c|c|c|c|}
\hline \multirow{2}{*}{\multicolumn{3}{|c|}{$\frac{\text { Visits }}{\text { Week(s) }}$}} & \multirow{2}{*}{$\frac{\text { Pre-screening }}{-}$} & \multirow{2}{*}{$\begin{array}{c}\text { Screening } \\
-\end{array}$} & \multirow{2}{*}{$\begin{array}{c}\text { T0 Baseline } \\
0 \\
\end{array}$} & \multicolumn{3}{|c|}{ Treatment: MoBa vs. CBT } & \multirow{2}{*}{$\begin{array}{c}\text { T1 Post } \\
16 \\
\end{array}$} & \multirow{2}{*}{$\begin{array}{c}\text { T2 Follow-up } \\
42 \\
\end{array}$} \\
\hline & & & & & & $1-4$ & $5-15$ & 16 & & \\
\hline \multirow{5}{*}{$\begin{array}{l}\frac{n}{n} \\
\frac{a}{a} \\
\frac{1}{\frac{1}{I}} \\
\frac{1}{I}\end{array}$} & Ses & ions per week & & & & 2 & 1 & 1 & & \\
\hline & \multirow{4}{*}{ 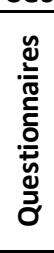 } & $\begin{array}{l}\text { Therapeutic } \\
\text { Element Checklist }\end{array}$ & & & & $x$ & $x$ & $x$ & & \\
\hline & & AE / SAE & & & & $x$ & $x$ & $x$ & & \\
\hline & & PHQ-9 & & & & $x$ & $x$ & $x$ & & \\
\hline & & WAI-P / WAI-T & & & & & & $x$ & & \\
\hline \multirow{16}{*}{$\frac{\tilde{\alpha}}{\frac{1}{\sigma}}$} & \multirow{5}{*}{ 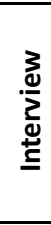 } & telephone screening & $(X)$ & & & & & & & \\
\hline & & SCID-5 (CV/PD) & & $x$ & & & & & & \\
\hline & & HRSD-24 & & $x$ & $(X)$ & & & & $x$ & $x$ \\
\hline & & SOFAS & & & $\mathrm{X}$ & & & & $\mathbf{x}$ & $\bar{x}$ \\
\hline & & $\mathrm{AE} / \mathrm{SAE}$ & & & $(X)$ & & & & $x$ & $x$ \\
\hline & \multirow{11}{*}{ 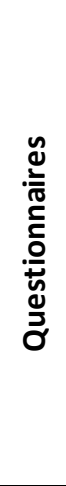 } & CTQ & & $x$ & & & & & & \\
\hline & & $\mathrm{RSQ}$ & & $x$ & & & & & $x$ & $x$ \\
\hline & & $\mid \mathrm{RI}$ & & $\mathrm{x}$ & & & & & $x$ & $x$ \\
\hline & & DERS-16 & & $x$ & & & & & $x$ & $x$ \\
\hline & & MZQ & & & $x$ & & & & $x$ & $x$ \\
\hline & & $\mathrm{BDI}-\mathrm{II}$ & & & $x$ & & & & $x$ & $x$ \\
\hline & & $\mathrm{BAl}$ & & & $x$ & & & & $x$ & $x$ \\
\hline & & WHOQoL-BREF & & & $x$ & & & & $x$ & $x$ \\
\hline & & PHQ-9 & & & $x$ & & & & $x$ & $x$ \\
\hline & & ECR-RD8 & & & $x$ & & & & $x$ & $x$ \\
\hline & & SBC & & & $x$ & & & & $x$ & $x$ \\
\hline
\end{tabular}


Figure 4: Decision Tree Algorithm for Modular-Based Psychotherapy.

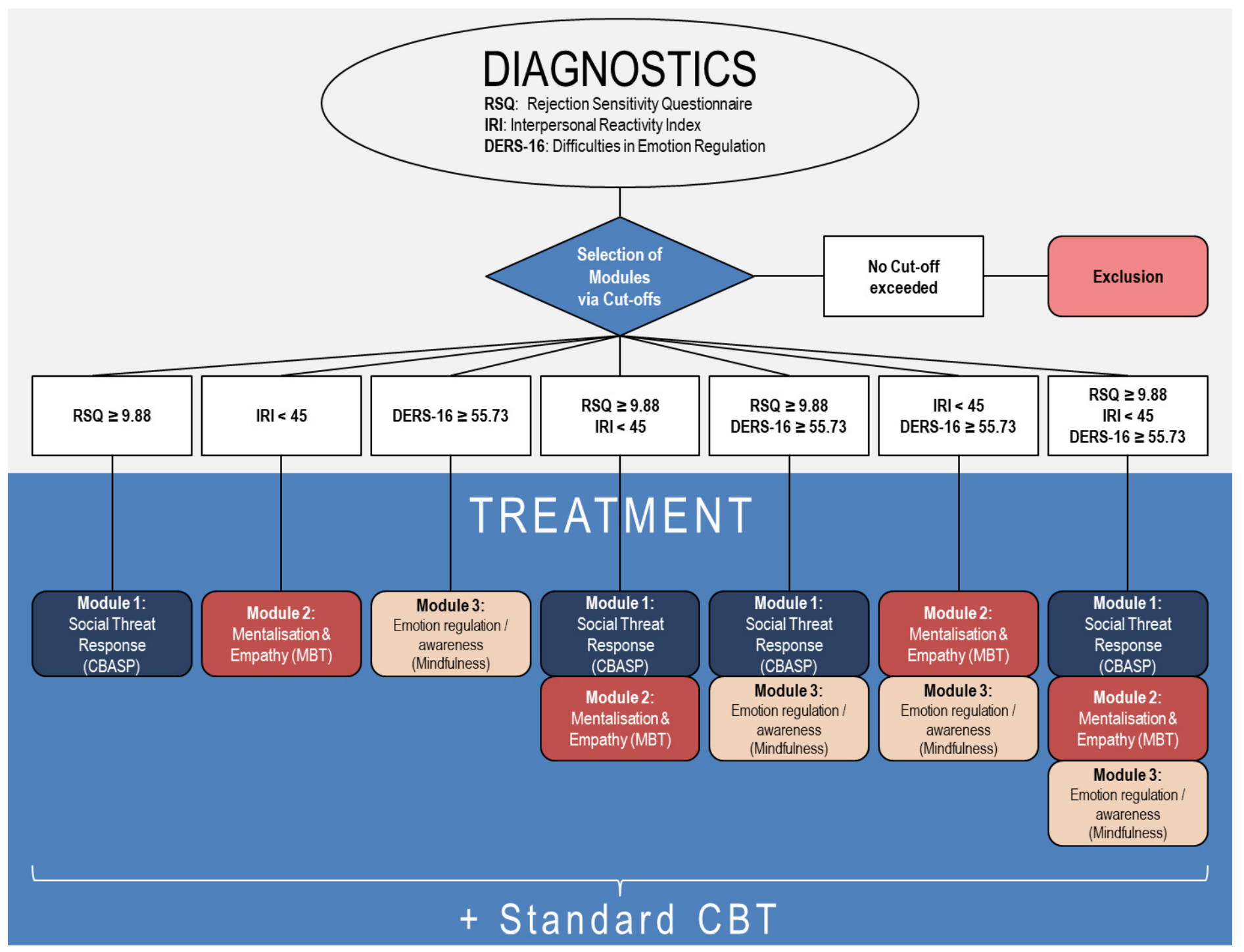


Table 1: Primary and secondary endpoints and corresponding measures.

\begin{tabular}{ll}
\hline \multicolumn{1}{c}{ Endpoint } & \multicolumn{1}{c}{ Measure } \\
\hline $\begin{array}{l}\text { Severity of } \\
\text { depression } \\
\text { (post treatment) }\end{array}$ & $\begin{array}{l}\text { Primary Endpoint: Hamilton Rating Scale for Depression (HRSD-24) [75] at the end of treatment } \\
\text { rated by trained and blinded clinicians. }\end{array}$ \\
\hline Feasibility & $\begin{array}{l}\text { Assessed by recruitment rates, distribution rates to the modules, and therapists' as well as } \\
\text { patients' ratings (Therapeutic Element Checklist; WAl-SR, [80]) }\end{array}$ \\
\hline $\begin{array}{l}\text { Severity of } \\
\text { depression (FUP) }\end{array}$ & HRSD-24 six months after end of treatment rated by trained and blinded clinicians. \\
\hline
\end{tabular}
questionnaire comprising 18 hypothetical interpersonal interactions with potential rejections by others (e.g. "You ask someone you don't know well out on a date"). It assesses the level of anxiety Social threat the patient feels about the outcome of each situation on a six point Likert scale ranging from "very response system unconcerned" to "very concerned". The RSQ shows good internal consistency and test-retest reliability, and is a reliable measure of the anxious-expectations-of-rejection component of rejection sensitivity. For the German version, the original has been translated, adapted, and shown to be a homogeneous measure with good psychometric properties [81].

Module questionnaire: The Interpersonal Reactivity Index (IRI) is a 28-item self-report instrument that measures both cognitive and emotional aspects of empathy. Items are rated on a five-point Likert scale ranging from 0 ("does not describe me well") to 4 ("describes me very well"). The questionnaire comprises 4 subscales (7 items each): Perspective Taking (e.g. "I sometimes find it difficult to see things from the 'other guys' point of view."), Fantasy (e.g. "I daydream and Mentalizing of fantasize, with some regularity, about things that might happen to me."), Empathic Concern (e.g. others' mental "I often have tender, concerned feelings for people less fortunate than me."), and Personal states / empathy Distress (e.g. "I sometimes feel helpless when I am in the middle of a very emotional situation."). The German version of the IRI [82] was reduced to only four items per scale and showed good psychometric properties.

The Mentalization Questionnaire (MZQ) [83] is a self-rating instrument for the assessment of mentalization in patients with mental disorders and consists of 15 items. The MZQ can be considered a practicable instrument with acceptable reliability and sufficient validity to assess mentalization in patients with mental disorders [83].

\begin{tabular}{|c|c|}
\hline $\begin{array}{l}\text { Emotion } \\
\text { awareness and } \\
\text { regulation }\end{array}$ & $\begin{array}{l}\text { Module questionnaire: A validated shorter version of the DERS [84,78] with } 16 \text { items. For each } \\
\text { of the DERS- } 16 \text { items, participants are asked to "indicate how much it applies to your emotions } \\
\text { right now" with response options ranging from } 1 \text { ("not at all") to } 5 \text { ("completely"). The questionnaire } \\
\text { has four subscales: Non-acceptance (i.e., non-acceptance of current emotions), Modulate (i.e., } \\
\text { difficulties modulating emotional and behavioural responses in the moment), Awareness (i.e., } \\
\text { limited awareness of current emotions), and Clarity (i.e., limited clarity about current emotions). } \\
\text { Results of the study provide support for the reliability and validity of the DERS-16 as a measure } \\
\text { of emotion regulation difficulties. }\end{array}$ \\
\hline $\begin{array}{l}\text { Response and } \\
\text { remission rates }\end{array}$ & $\begin{array}{l}\text { Response is defined as a reduction in the HRSD- } 24 \text { score by at least } 50 \% \text { from baseline and a } \\
\text { total score of less than 16; remission is defined a priori as an HRSD- } 24 \text { score of } \leq 8 \text {. }\end{array}$ \\
\hline $\begin{array}{l}\text { Social and } \\
\text { Occupational } \\
\text { Functioning }\end{array}$ & $\begin{array}{l}\text { The clinician-rated Social and Occupational Functioning Assessment Scale (SOFAS) [85] } \\
\text { assesses social role functioning irrespective of psychopathology. }\end{array}$ \\
\hline Quality of Life & $\begin{array}{l}\text { The WHO Quality of Life Instrument (WHOQOL-BREF) [86] is a short form tool consisting of } 26 \\
\text { items divided into } 4 \text { domains (physical health, psychological health, social relationships, and the } \\
\text { environment) to measure quality of life. }\end{array}$ \\
\hline $\begin{array}{l}\text { Self-rated } \\
\text { depressive and } \\
\text { anxiety symptoms }\end{array}$ & $\begin{array}{l}\text { Self-ratings of depressive and anxiety symptoms will be obtained using the Beck Depression } \\
\text { Inventory (BDI-II) [87] and the Beck Anxiety Inventory (BAI) [88]. }\end{array}$ \\
\hline $\begin{array}{l}\text { Body } \\
\text { connectedness }\end{array}$ & $\begin{array}{l}\text { Self-ratings of body awareness and bodily dissociation will be obtained using the Scale of Body } \\
\text { Connectedness (SBC) [90]. }\end{array}$ \\
\hline $\begin{array}{l}\text { Therapeutic } \\
\text { alliance }\end{array}$ & $\begin{array}{l}\text { The Working Alliance Inventory-Short Revised (WAI-SR) [80] assesses three key aspects of the } \\
\text { therapeutic alliance: (a) agreement on the tasks of therapy, (b) agreement on the goals of therapy } \\
\text { and (c) development of an affective bond. }\end{array}$ \\
\hline $\begin{array}{l}\text { Course of } \\
\text { depressive } \\
\text { symptoms }\end{array}$ & $\begin{array}{l}\text { Patients will fill out the Patient Health Questionnaire-9 (PHQ-9) [91] before every session to } \\
\text { constantly monitor depressive symptom severity as a proxy of therapy progress or deterioration. }\end{array}$ \\
\hline $\begin{array}{l}\text { Therapeutic } \\
\text { Element Checklist }\end{array}$ & $\begin{array}{l}\text { All elements/strategies/components will be recorded immediately after each session including the } \\
\text { approximate time the therapist used for applying those interventions using a Therapeutic Element } \\
\text { Checklist designed for this feasibility trial. }\end{array}$ \\
\hline
\end{tabular}




\section{CBASP. \\ Module}

Corresponding RDoC domain: Negative Valence System: Social Threat Response

Indicative questionnaire: $\quad$ Rejection Sensitivity Questionnaire (RSQ)

Objective:

"Re-training" the negative valence system (social threat response) and reducing avoidance behaviour called "Significant Other History" ( $\mathrm{SOH}$ ) is conducted, a short procedure listing significant others who left an interpersonal-emotional "stamp" in the patient's learning history. From the SOH, causa conclusions are derived (e.g. "Growing up with my mother led to the pervasive assumption that I have nothing to expect from others"). Based on the patient's assumptions about relationships the patient experienced in his/her history with abusive significant others, a proactive "transference hypothesis" is formulated stating the patient's most relevant interpersonal expectation/fear regarding the therapist-patient encounter The transference hypothesis is then systematically contrasted with the therapist's actual behaviour in "hot spot situations", applying the structured "Interpersonal Discrimination Exercise". By means of this exposure procedure, the patient learns to differentiate the abusive significant other (generalized to his/her social environment) from current non-abusive or well-intended persons by discrimination learning. Thus, the patient is enabled to overlearn dysfunctional expectations and reprogram the conditioned social threat systems. In addition, by enriching safety signals in therapists' behaviour and re-establishing the perception of operant interpersonal contingencies, this intervention is designed to provide a secure learning environment to decrease interpersonal threat sensitivity. In addition, teaching the patient the mechanisms of complementary interpersonal processes illustrated by Kiesler's circumplex model [92] enables the patient to recognize the consequences of his/her own behaviour on other persons and to develop empathy ("reading others") and social problem-solving skills (element of CBASP). Genuine empathy and theory of mind skills are furthermore facilitated by the therapist's "Disciplined Personal Involvement" (DPI) and more specifically "Contingent Personal Reactivity" (CPR), i.e. expressing personal emotional reactions to the patients' dysfunctional behaviour patterns in a disciplined way (including considering a teachable moment and relating it to the patient's core pathology) and offering alternative behaviour. The key objective of this module is social fear extinction by overlearning conditioned associations and avoidance behaviour.

\section{Mentalising-} Module

Corresponding RDoC domain: System for Social Processes

\section{Indicative questionnaire: Interpersonal Reactivity Index (IRI)}

Objective:

The Mentalising-Module contains modelling and teaching mentalising by learning to "read" others' behaviour and thereby re-connecting the patient to his/her social environment and creating socia competence. To promote mentalised affectivity (i.e. mentalising own emotional states as described by MBT), the therapist introduces repetitive sequences to stimulate basic mentalising functions in the patient. Based on empathy, the therapist uses a "not knowing" stance of exploration of the patients' experiences and identifies context-related emotional reactions, raising "what-questions" rather than "why-questions". Two typical interventions to engage mentalising are the "Stop and Stand" and the "Stop, Re-wind, Explore" sequences [67]. In the first case, the therapist stops a patient who is stuck in drawing non-mentalising assumptions (e.g. "everybody hates me") by surprise or humour to subsequently help the patient to mentalise about his/her experiences. The second sequence generates a joined attention on the patient's past experiences by shifting the focus back and forth within an episodic experience to make it accessible for the mentalising process. Genuine empathy and theory of mind skills are furthermore facilitated by the therapist's "Disciplined Personal Involvement" (DPI; and more specifically "Contingent Personal Reactivity" (CPR) as an element of CBASP as well. The key objective of this module is to improve mentalising capabilities in social interactions.

\section{Mindfulness- Module \\ Corresponding RDoC domain: Indicative questionnaire:}

Objective:

Arousal System: Hyperarousal

Difficulties in Emotion Regulation (DERS-16)

Reducing the arousal system (hyperarousal) referring to emotion awareness and regulation

This module integrates mindfulness-based exercises, which focus on a) observing non-judgmentally internal and external stimuli, b) shifting attention away from trauma-related inner "movies" and monitoring skills to c) overcome hyperarousal and experiential avoidance or being run over by one's emotions. Mindfulness-based interventions aim to change a person's perspective on his or her emotions and cognitions. This process is facilitated through mindfulness meditation (e.g., body scan, formal sitting meditation) in which close attention is paid to the present moment whilst thoughts, feelings and body sensations are noted with an attitude of curiosity, non-judgement, and acceptance of psychological experiences. Mindfulness has been suggested to be effective via four mechanisms: attention regulation, body awareness, changes in perspective on the self, and emotion regulation [93,94]. Mindfulness training enhances positive affect [95], decreases negative affect, and reduces maladaptive automatic emotional responses [96] being associated with changes in areas of the brain responsible for affect regulation and stress impulse reaction [97,93]. The key objective of this module is to improve emotion awareness and regulation in order to mitigate hyperarousal. 